\title{
The Argonne Target Library
}

\author{
Gavin Gordon ${ }^{1}$, Matthew Gott ${ }^{1}$, and John P. Greene ${ }^{1, *}$ \\ ${ }^{1}$ Physics Division, A rgonne National Laboratory, 9700 South Cass A venue, Lemont, IL, 60439, U nited States
}

\begin{abstract}
As part of the proposal to DOE-NP for the Center for Accelerator Target Science (CATS) initiative, one of the objectives was to develop an inventory of existing targets that will serve as a pool available to the community. Targets collections have been recovered from $Y$ ale University due to the closing of their Tandem A ccelerator Facility. In addition, accumulated targets from target preparation in the Physics Division over several decades have also been assembled with the intent of providing them to whomever would have a use for them. Space has now become available to compile, catalogue and house these collections. Thus, the A rgonne Target Library has been established and its progress and outlook will be discussed in detail.
\end{abstract}

\section{Introduction and motivation}

\subsection{Center for Accelerator Target Science (CATS)}

Over the past decade, the need for targets for the lowenergy nuclear physics program has been expressed in many venues. With the closure of several accelerator laboratories, the demand for targets has only increased on existing target-making facilities. The Physics Division at Argonne National Laboratory (ANL) maintains a target development laboratory in direct support of ongoing low-energy nuclear physics research undertaken at the Argonne Tandem Linac Accelerator System (ATLAS) facility. The mission of the target laboratory is also to support research by the low-energy research group of the Physics Division. Under certain conditions, the target laboratory has also supplied targets to other scientists, including ATLAS users, for experiments carried out at other locations, both within the US and abroad, but only a fraction of these outside requests can be addressed. It was thus proposed to create a National Center for Accelerator Target Science (CATS) based on the existing target development laboratory at ANL. The objectives of the center are as follows:

1. Serve the low-energy community by producing targets whenever possible by either manufacturing them or by directing requests to other facilities best able to perform the task;

2. Train individual investigators and students in target making in order to provide a workforce capable to address present and future needs;

3. Carry out $R \& D$ activities dedicated to novel production techniques and optimization of existing ones;
4. Develop an inventory of existing targets that will serve as a pool available to the community.

Over the past several decades, a very large number of targets have been produced and could possibly be reused if they were easy to locate. Sletten, in 1974, described a target catalogue system and library used at the Niels Bohr Institute [1]. This CATS proposal would explore the feasibility of generating an inventory of targets from several institutions and made available for reuse.

\subsection{The closing of the Yale Tandem}

Upon the closure of the Wright Nuclear Structure Laboratory (WNSL) at Y ale University [2] there arose the need to preserve many of the target collections already housed there. Targets from both Brookhaven National Laboratory (BNL) as well as Los Alamos National Laboratory had been stored and cataloged at WNSL, occupying several cabinets. These, along with the numerous targets prepared over the years, were recovered and shipped to $A N L$ as part of the initial target library collections.

\subsection{The Physic Division Target collection}

W hile the targets collected from $Y$ ale have constituted $a$ good start to the A rgonne Target Library, there exists an accumulation of accelerator targets prepared by the Physics Division Target L aboratory. Over a time span of thirty plus years these targets have been in use, reuse and just stored for eventual disposal. Now, with the advent of the target library, space is available for sorting and cataloging this large collection of targets for reuse by others. The size of this collection has resulted in an ongoing effort which is still underway.

\footnotetext{
* Corresponding author: greene@ anl.gov
} 


\section{The Argonne Target Library}

Work has begun assembling this inventory of existing targets that will serve as a pool available to the lowenergy nuclear physics community on a first-come firstserve basis. Target collections stored at $Y$ ale U niversity consisting of targets recovered from BNL and $L A N L$, targets prepared at $\mathrm{Y}$ ale and added to those already existing at $A N L$ will form the initial holdings of the A rgonne Target Library.

\subsection{Space establish}

First needed would be a space large enough for the shelves and cabinets needed to hold the collections with some thought as to future space for additional target collections as they might arise. With generous assistance for the A rgonne Physics Division a large room, formerly part of the 6-M eV V an de Graff accelerator facility, was made available for this purpose. The area required decommissioning and extensive clean-up. With the equipment and storage removed from the room, the floor was resealed and the windows and blinds cleaned. A $n$ office desk and laboratory bench were not discarded and instead kept for eventual library use.

\subsection{Furnishings found}

Throughout the Physics Division Building can be found spare furnishings and shelve units available for re-use. Several storage cabinets were also located and moved into the newly recovered space. A schematic of the Target Library layout is shown in Fig. 1. Additional furnishings consisting of a computer desk and office chairs, etc. were quickly assembled. A network line was needed and desktop computer needed for target listings was installed.

\subsection{The Physics Division Target Archives}

Ancillary to the Target Library and therefore co-located within are the Physics Division Target Archives. The totality of target preparation notes dating back to 1963 constitute these archives. Over the years these collections of target preparation notes have been stored in various locations within the Physics Division, including the basement. Every target job and accompanying writings are held in a series of binders containing the target request and notes detailing the target preparation techniques employed, including information on isotope and assays [3]. A fter 1978 these target request pages have been digitized and are now contained in a searchable database for instant look-up. The target job information can then be easily found in these collections of binders by J ob N umber. It is natural for this collection to therefore find a home in the Target Library.

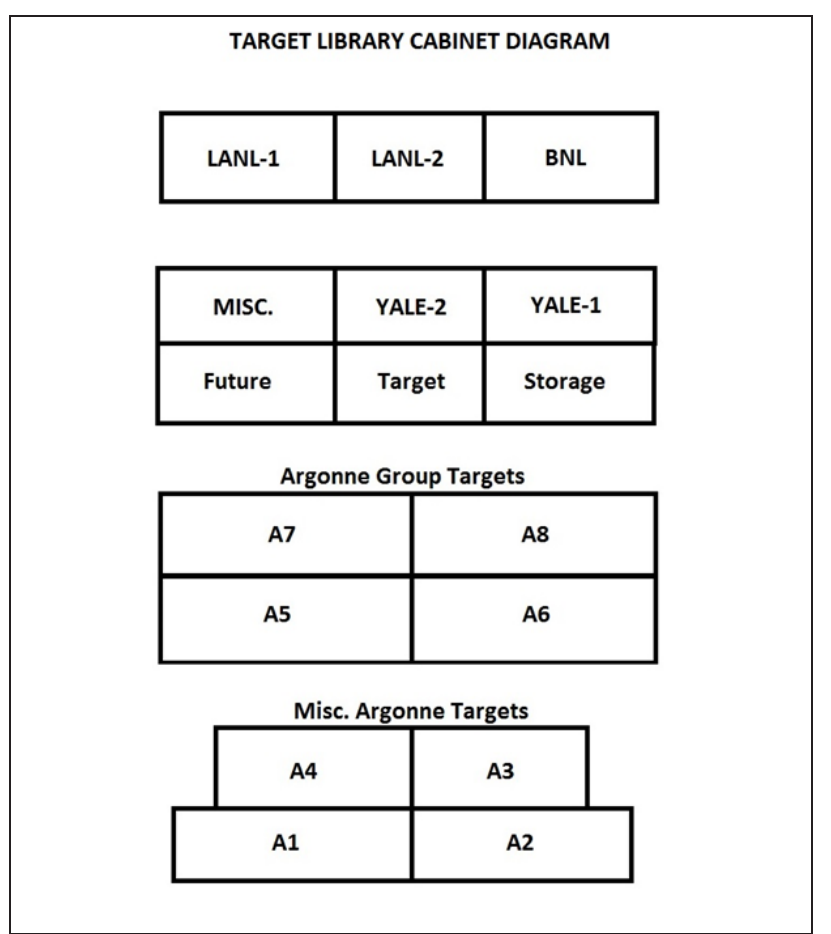

Fig. 1. Schematic layout of the A rgonne Target L ibrary

\section{The collections}

We describe here now the many collections of targets which will constitute the newly established Argonne Target Library.

\subsection{The Wright Nuclear Structure Laboratory}

As mentioned previously, the Wright Nuclear Structure Laboratory at $Y$ ale U niversity had inherited collections of targets from both LANL and BNL when their respective low-energy nuclear physics programs were discontinued. With the closing of the WSNL these individually packaged targets, extensively catalogued, listed in .pdf files (see for example Fig. 2) and adequately stored in a series a of cabinets were carefully packed and shipped to ANL. It was a simple procedure to unpack and reshelf these two collections into the cabinets installed in the target library.

\subsection{The Physics Division Target Laboratory}

Encompassing a span of over thirty years, many target preparations have been performed within the Physics Division Target Laboratory which resulted in many hundreds of targets made during practice depositions as well as actual targets whereby many are produced to make efficient use of precious separated isotope. This results in many unused targets available for re-use via the target library. They have been stored over the years in the target lab and at various locations within the ATLAS facility. 


\begin{tabular}{|c|c|c|c|c|}
\hline \multicolumn{5}{|l|}{ Stand A } \\
\hline Number & Element & Mass number & Thickness & Backing \\
\hline 1 & LiF & & 200 & $30 \mathrm{C}$ \\
\hline 2 & c & & 850 & : \\
\hline 3 & $\begin{array}{l}\text { c } \\
\text { c }\end{array}$ & & $\begin{array}{l}20 \\
20\end{array}$ & : \\
\hline $\begin{array}{l}4 \\
5\end{array}$ & $\begin{array}{l}c \\
c\end{array}$ & & 20 & : \\
\hline $\begin{array}{l}5 \\
6\end{array}$ & $\begin{array}{l}\mathrm{C} \\
\mathrm{c}\end{array}$ & 12 & $\sim 50$ & : \\
\hline $\begin{array}{l}6 \\
7\end{array}$ & c & 12 & $\begin{array}{c}10 \\
40-50\end{array}$ & : \\
\hline 8 & $\mathrm{~N}$ & 15 & $\begin{array}{l}40-50 \\
100\end{array}$ & $10 \mathrm{C}$ \\
\hline 9 & Mg & 26 & -200 & \\
\hline 10 & Mg & 26 & 10 & $20 \mathrm{C}+1 \mathrm{Bi}$ \\
\hline 11 & $\mathrm{Mg}$ & 26 & 10 & $20 \mathrm{C}+1 \mathrm{Bi}$ \\
\hline 12 & $\mathrm{Mg}$ & 26 & 10 & $20 \mathrm{C}+1 \mathrm{Bi}$ \\
\hline 13 & Al & & 75 & Polystyrene, overcoat $10 \mathrm{Au}$ \\
\hline 14 & Al & Pure & 400 & Crème Cote \\
\hline 15 & AI & Pure & 400 & Creme Cote \\
\hline 16 & Al & & 815 & 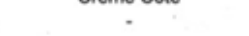 \\
\hline 17 & Al & & 10 & . \\
\hline 18 & Al & & 1 & . \\
\hline 19 & AI & Spot & 100 & $20 \mathrm{C}$ \\
\hline 20 & Al & & 20 & overcoat $10 \mathrm{Au}$ \\
\hline 21 & A & & 75 & overcoat $10 \mathrm{Au}$ \\
\hline 22 & Al & & 450 & (1) \\
\hline 23 & Al & & 450 & $2 \mathrm{Au}$ \\
\hline 24 & Al & Pure & 400 & Crème Cote \\
\hline 25 & Al & & 160 & . \\
\hline 26 & A) & Spot & 81 & $20 \mathrm{C}$ \\
\hline 27 & Al & & 1 & - \\
\hline 28 & $\mathrm{Si}$ & 28 & 10 & $20 \mathrm{C}$ \\
\hline 29 & $\mathrm{si}$ & 28 & 10 & $20 \mathrm{C}$ \\
\hline 30 & $\mathrm{SiO}_{2}$ & & 150 & - \\
\hline 31 & $\mathrm{Ca}$ & 48 & 98 & $20 \mathrm{C}$ \\
\hline 32 & Sc & & 3.85 & \\
\hline 33 & $\pi$ & Nat & 500 & $5 \mathrm{mil} \mathrm{Ta}$ \\
\hline 34 & $\pi$ & Nat & 500 & $5 \mathrm{mill} \mathrm{Ta}$ \\
\hline 35 & $\pi$ & 46 & 1.02 & $\mathrm{~F}=4 \mathrm{~cm}^{2}$ \\
\hline 36 & $\pi$ & 48 & 1.04 & $\mathrm{~F}=3 \mathrm{~cm}^{2}$ \\
\hline 37 & $\pi$ & 50 & 1 & $\mathrm{~F}=3 \mathrm{~cm}^{2}$ \\
\hline 38 & Mn & Nat & 108 & Polystyrene \\
\hline 39 & $\mathrm{Fe}_{2} \mathrm{O}_{3}$ & Nat & 36 & $20 \mathrm{C}$ \\
\hline 40 & co & 59 & 23 & $20 \mathrm{C}$ \\
\hline 41 & co & & 62 & $20 \mathrm{C}$ \\
\hline 42 & co & 59 & 28 & $20 \mathrm{C}$ \\
\hline 43 & co & 59 & 28 & $20 \mathrm{C}$ \\
\hline 44 & Co & & 23 & $20 \mathrm{C}$ \\
\hline 45 & Ni & 60 & 50 & $20 \mathrm{C}$ \\
\hline 46 & $\mathrm{Ni}$ & & $0.00002^{2}$ & \\
\hline 47 & Ni & 60 & 50 & $5-10 \mathrm{C}$ \\
\hline
\end{tabular}

Fig. 2. Example page from the list of BNL targets

\subsection{Various miscellaneous collections}

In addition to the targets described above, there are many additional targets which have been brought or shipped to Argonne for experiments at ATLAS. Plus there have been many targets produced for Physics Division employees conducting experiments at other facilities throughout the world. These targets are characterized by their originating institution as well as the unique target frames employed. These smaller assemblies of targets were simply entered into the computer as individual lists using M icrosoft W ord.

\section{Current progress and outlook}

M uch progress has already been made but there is still some unknowns to be determined and work finished up. While the $L A N L$ and $B N L$ targets were already nicely catalogued the various other target collections ( $Y$ ale U., $A N L$, etc.) and their databases will need to be formulated and built. In the end a working distribution system will need to be established with some simple rules applied. Widespread advertisement and target availability will be accomplished by this publication as well as word-of-mouth through the INTDS and access from the newly established CATS W ebsite.

\subsection{Reconstituting the Yale targets}

In addition to the carefully listed targets from $L A N L$ and $B N L$ recovered from $W N S L$, there were many targets prepared at $Y$ ale over the years and when the lab was closed down, these targets were carefully boxed up and shipped back to ANL. The database chosen for target cataloguing was Microsoft Access which is what is currently employed for the Physics Division Target A rchives. Also unexpectedly arrived from $Y$ ale were collections of target preparation materials organized by element.

\subsection{Cataloguing the ATLAS collections}

The various collections of targets accumulated throughout ATLAS and the Physics Division constitute the largest amount of targets to be considered. Here we find many boxes of individual targets as well as groups of targets by experiment, apparatus and investigator. We found it prudent to keep these groups intact and have chosen M icrosoft Excel as the simplest way to list these targets. The individual targets were sorted and entered into the computer using Microsoft Access for alphabetical listing.

\subsection{Target library operations and CATS website}

Once all the various target catalogues are completed, target library operations can commence. So as to prevent individual monopolizing of library requests we are proposing a set of modest rules to be followed:

1. Individual target requests will be limited to one specified target and up to two targets shipped (if possible \pm for a spare).

2. Priority will be assigned to domestic requests and of those, being DOE ( $\&$ NSF) investigators will be addressed first.

3. Wherever possible a user supplied FedEx account will be used for shipping.

4. When necessary, if a vacuum target transport container [4] is needed (such as those marketed by M icroFoils, Inc.) \pm It should be supplied in advance by the requestor.

5. Additional considered rules which may present themselves in the future could be approved as necessary.

These library rules and target collection listings will be made available in the near future via the Physics Division CATS W ebsite.

\section{Conclusion}

In conclusion, the Argonne Target Library is now coming online. Once the catalogued collections are being compiled and completed, they should become rapidly available through a link from the CA TS website. We anticipate a slow and steady demand for these targets once established. The mechanism for target check-out 
should quickly be realized once underway. Hopefully, this vast wealth of unused targets may again become available to the low-energy community for use in nuclear physics experiments.

This material is based upon work supported by the U.S. Department of Energy, Office of Science, Office of Nuclear Physics, under Contract No. DE-AC02-06CH11357. This research used resources of ANL'S ATLAS facility, which is a DOE Office of Science U ser Facility. The authors are grateful to the site planning and area clean-up provided by Kimberly Miller, furniture procurement from Building $M$ anager $K$ eith Trychta and janitorial support from D avid Streeter.

\section{References}

1. G. Sletten, INTDS Conference Proceedings 47 (1974)

2. http://www.yalescientific.org/2015/03/rebirth-ofwright

3. G.E. Thomas, J.P. Greene, N ucl. Instr. M eth. Phys. Res. A 362, 201 (1995)

4. J.H. B jerregaard, P. K nudsen, G. Sletten, B ook, Ed. : J. J aklovsky, Plenum Press, NY , 207 (1981) 